\title{
TWO-TERM EDGEWORTH EXPANSION FOR M-ESTIMATORS OF A LINEAR REGRESSION PARAMETER WITHOUT CRAMÉR-TYPE CONDITIONS AND AN APPLICATION TO THE BOOTSTRAP
}

\author{
I. KARABULUT and S. N. LAHIRI
}

(Received 24 January 1996; revised 4 July 1996)

Communicated by A. G. Pakes

\begin{abstract}
A two-term Edgeworth expansion for the distribution of an M-estimator of a simple linear regression parameter is obtained without assuming any Cramer-type conditions. As an application, it is shown that certain modification of the naive bootstrap procedure is second order correct even when the error variables have a lattice distribution. This is in marked contrast with the results of Singh on the sample mean of independent and identically distributed random variables.
\end{abstract}

1991 Mathematics subject classification (Amer. Math. Soc.): primary 62F12; secondary $62 \mathrm{~J} 05$.

Keywords and phrases: Bootstrap, Cramér's condition, Edgeworth expansion, M-estimators, regression.

\section{Introduction}

The following simple regression model will be considered throughout this paper

$$
Y_{j}=x_{j} \beta+\epsilon_{j}, \quad j=1,2, \ldots, n,
$$

where $\epsilon_{1}, \epsilon_{2}, \ldots, \epsilon_{n}$ are independent and identically distributed (i.i.d.) random variables (r.v. 's) with a common distribution function (d.f.) $F, \beta$ is the unknown regression parameter to be estimated, and $x_{1}, x_{2}, \ldots, x_{n}$ are known non-random constants. In the sequel some restrictions will be imposed on these constants in order to obtain the results of this paper. Let $\psi$ be a nondecreasing function on the real line $\mathbb{R}$, satisfying the assumption

$$
E \psi\left(\epsilon_{1}\right)=0 .
$$

The work of the first author done at Iowa State University.

(C) 1997 Australian Mathematical Society 0263-6115/97\$A2.00+0.00 
An M-estimator $\bar{\beta}_{n}$ of $\beta$ corresponding to $\psi$ is defined to be a solution of the equation (cf. Huber [8])

$$
\sum_{j=1}^{n} x_{j} \psi\left(Y_{j}-x_{j} t\right)=0 .
$$

In particular, $\psi(x) \equiv x$ yields the least square estimator (LSE) of $\beta$. Consistency and asymptotic normality of $\bar{\beta}_{n}$ has been established by Huber [8] in a more general setting. More accurate approximations for the distribution of $\bar{\beta}_{n}$ can be obtained using Edgeworth expansions. In recent years, there have been a number of studies on Edgeworth expansion for $\bar{\beta}_{n}$, viz. Ringland [13], Navidi [11], Qumsiyeh [12], Tiro [16] and Lahiri [10]. In all these works excepting Navidi [11], it is assumed that the distribution of $\psi\left(\epsilon_{1}\right)$ satisfies some variant of the following smoothness condition, known as the Cramér condition:

For every $\delta>0$, there exists a $0<q<1$ such that $\mid E \exp \left(\right.$ it $\left.\psi\left(\epsilon_{1}\right)\right) \mid<q$

$$
\text { for all }|t|>\delta \text {. }
$$

Navidi [11] uses a weaker condition, requiring only that $\psi\left(\epsilon_{1}\right)$ be non-lattice. (A r.v. $W$ is called non-lattice if $|E \exp (i t W)| \neq 1$ for all $t \neq 0$; otherwise, it is called a lattice r.v. See Feller [5] for more details.) Note that the non-lattice and the Cramér conditions both hold when the distribution of $\psi\left(\epsilon_{1}\right)$ has a density. However, there are many important discrete error distributions for which $\psi\left(\epsilon_{1}\right)$ fails to satisfy either of the two smoothness conditions. In this paper, we provide appropriate conditions on the design points $x_{i}$ 's such that two-term Edgeworth expansions hold for standardized M-estimators irrespective of such smoothness of $\psi\left(\epsilon_{1}\right)$. Consequently, one can use our result to obtain a more accurate approximation to the distribution of standardized M-estimators (including the LSE) without having to add separate continuity correction terms when $\psi\left(\epsilon_{1}\right)$ is a lattice r.v.

Next, we consider bootstrapping $\bar{\beta}_{n}$ under model (1.1). Let $\epsilon_{1}^{*}, \ldots, \epsilon_{n}^{*}$ denote a random sample from the residuals

$$
\widehat{\epsilon}_{j}=Y_{j}-x_{j} \bar{\beta}_{n}, \quad j=1,2, \ldots, n .
$$

Then, the 'naive' bootstrap estimator $\beta_{n}^{*}$ of $\bar{\beta}_{n}$ is defined as a solution to the equation (cf. Efron [4])

$$
\sum_{j=1}^{n} x_{j} \psi\left(Y_{j}^{*}-x t\right)=0
$$

where $Y_{j}^{*}$ 's denote the bootstrap observations, defined by $Y_{j}^{*}=x_{j} \bar{\beta}_{n}+\epsilon_{j}^{*}, 1 \leq j \leq n$. This naive approach of bootstrapping M-estimators poses a serious problem under model (1.1), which has been detected by Freedman [6] for the LSE, and by Shorack 
[14] and Lahiri [10] for general M-estimators. Unless the bootstrap resamples are selected appropriately to ensure the bootstrap analogue of (1.2), viz.,

$$
E_{n} \psi\left(\epsilon_{1}^{*}\right)=0,
$$

the limit distribution of $a_{n}\left(\beta_{n}^{*}-\bar{\beta}_{n}\right)$ incorporates a random bias term and fails to approximate the distribution of $a_{n}\left(\bar{\beta}_{n}-\beta\right)$. (Here, $a_{n}^{2}=\sum_{j=1}^{n} x_{j}^{2}$, and $E_{n}$ denotes the conditional expectation given $Y_{1}, \ldots, Y_{n}$.) Since (1.4) does not hold for the naive bootstrap approach described above, it fails under model (1.1). Two modifications of the naive bootstrap for M-estimators have been suggested by Shorack [14] and Lahiri [10]; these can be described as 'centering the score function $\psi$ ' and 'changing the resampling distribution' respectively. Here, we will employ the second one where one draws i.i.d. bootstrap observations $\epsilon_{1}^{*}, \ldots, \epsilon_{n}^{*}$ from the weighted empirical distribution

$$
F_{n}(y)=p_{n}^{-1} \sum\left|x_{j}\right| I\left(\epsilon_{j}^{*} \leq y\right),
$$

putting mass $\left|x_{j}\right| / p_{n}$ at $\widehat{\epsilon}_{j} j=1,2,3, \ldots, n$, where $p_{n}=\sum_{j=1}^{n}\left|x_{j}\right|$. Note that when this weighted empirical distribution has been employed and $x_{j}$ 's are all positive or all negative, then the required assumption (1.4) is met.

Asymptotic validity of the modified bootstrap procedures have been established by Freedman [6] and Shorack [14], and the issue of second order superiority have been investigated by Navidi [11], Qumsiyeh [12], Tiro [16] and Lahiri [10] under different sets of regularity conditions on the design constants $x_{i}$ 's, the score function $\psi$ and the unknown error distribution $F$. As in the case of deriving Edgeworth expansions, all these works on the second order accuracy of the bootstrap require the 'non-lattice' condition on the r.v. $\psi\left(\epsilon_{1}\right)$. From the seminal work of Singh [15] it is well known that for the sample mean of iid r.v. s, (which is a special case of our model with $x_{i} \equiv 1$ and $\psi(x) \equiv x)$, the bootstrap approximation is second order correct if and only if the underlying distribution $F$ is non-lattice. In contrast, we show that this is not a necessary condition for the second order correctness of the bootstrap under the full generality of model (1.1). This is particularly important from an application point of view, since most of the standard discrete distributions are lattice distributions.

The rest of the paper is organized as follows. In Section 2, we state the assumptions and the main results. In Section 3, we give the proofs.

\section{Assumptions and results}

Before stating the results, we set up the notation. Define $a_{n}^{2}=\sum_{j=1}^{n} x_{j}^{2}$, and $M_{n}=\max \left\{\left|x_{j}\right|: 1 \leq j \leq n\right\}$. For $x \in \mathbb{R}$, write $\mu(x)=E \psi\left(\epsilon_{1}-x\right), V(x)=\sigma^{2}(x)=$ $\operatorname{Var}\left(\psi\left(\epsilon_{1}-x\right)\right), \mu_{3}(x)=E\left(\psi\left(\epsilon_{1}-x\right)-\mu(x)\right)^{3}$, and $\rho_{3}(x)=E\left|\psi\left(\epsilon_{1}-x\right)-\mu(x)\right|^{3}$. 
The first three derivatives of a function $g$ defined on $\mathbb{R}$ will be denoted by $g^{\prime}, g^{\prime \prime}$, and $g^{\prime \prime \prime}$, respectively. Furthermore, define $A=-\mu^{\prime}(0) / \sigma(0), d_{1 n}=\sum_{j=1}^{n} x_{j}^{3} / a_{n}^{3}$, $d_{3 n}=\sum_{j=1}^{n}\left|x_{j}\right|^{3} / a_{n}^{3}, d_{2 n}=\sum_{j=1}^{n} x_{j}^{4} / a_{n}^{4}$ and let $b_{n}=\log a_{n}$, whenever it is defined. For the moment deferring the specification of the $r_{n}$ 's, let $C_{n}$ be the set of integers given by

$$
C_{n}=\left\{1 \leq j \leq n:\left|x_{j}\right| \leq d_{3 n} a_{n} / r_{n}\right\} .
$$

We are now ready to list the assumptions in addition to the ones given heretofore. We have two groups of assumptions. The first group of assumptions are required for both Edgeworth expansion and bootstrap approximation. They are labelled (A.1) through (A.4). The second group of assumptions are labelled as (R.1) and (R.2). The assumption (R.2) is used in Theorem 2.2, in the context of the bootstrap approximation.

(A.1) $a_{n} \rightarrow \infty$ as $n \rightarrow \infty$.

(A.2) There exist a sequence $\left\{r_{n}\right\}$ such that $r_{n} \rightarrow \infty$ as $n \rightarrow \infty$ and $\sum_{j \in C_{n}} x_{j}^{2} /\left(d_{3 n}^{2} a_{n}^{2}\right) \rightarrow \infty$.

(A.3) $A>0$ whenever it exists.

(A.4) $M_{n} b_{n}=o\left(a_{n}\right)$ as $n \rightarrow \infty$.

REMARK 2.1. Conditions (A.1) and (A.3) are required for the asymptotic normality of the M-estimator $\bar{\beta}_{n}$ (cf. Huber [8])). Typically $a_{n}$ is of the order $\sqrt{n}$. Condition (A.4) is used here to express the Edgeworth expansion for $\bar{\beta}_{n}$ in a simple form (cf. Theorem 2.1). Note that under (A.4), $M_{n} / a_{n}=o(1)$ as $n \rightarrow \infty$. In this study, Condition (A.2) has a distinctive position and, it is this assumption that makes possible the Edgeworth expansion and the bootstrap approximation without requiring any Cramér-type conditions. Note that, since $\sum_{j \in C_{n}} x_{j}^{2} /\left(d_{3 n}^{2} a_{n}^{2}\right) \rightarrow \infty$ as $n \rightarrow \infty$, any choice of $r_{n}$ must satisfy $r_{n} /\left|C_{n}\right| M_{n} \rightarrow 0$ as $n \rightarrow \infty$, where $\left|C_{n}\right|$ denotes the number of elements in the set $C_{n}$. For instance, if $x_{j}$ are given by $x_{j}=j$, then the assumption is viable. For this example, $r_{n}$ may be taken as $n^{1 / 4}$.

Next we list the other assumptions.

(R.1) (i) The function $\mu_{i}(x)$ has $3-i$ continuous derivatives in a neighborhood $N$ of zero, $i=0,1,2$, and $\mu_{3}$ is continuously differentiable in the neighborhood $N$.

(ii) There exists an $\alpha>0$ such that $E\left|\psi\left(\epsilon_{1}+\alpha\right)\right|^{3}<\infty$.

(R.2) $\psi$ has uniformly continuous bounded derivatives $\psi^{(k)}$ for $k=0,1,2$.

The results of this paper are given below.

THEOREM 2.1. Assume that Conditions (A.1) - (A.4) and (R.1) are satisfied. Then,

$$
\sup _{y \in \mathbb{R}}\left|P\left(a_{n} A\left(\bar{\beta}_{n}-\beta\right) \leq y\right)-\xi_{3 n}(y)\right|=o\left(d_{3 n}\right)
$$


Here $\xi_{3 n}(y)$, the Edgeworth expansion for the distribution function of $a_{n} A\left(\bar{\beta}_{n}-\beta\right)$ is given by

$$
\xi_{3 n}(y)=\Phi(y)-d_{1 n}\left[\left(\frac{\mu^{\prime \prime}(0)}{\sigma(0)}-\frac{\mu^{\prime}(0) V^{\prime}(0)}{\sigma^{3}(0)}\right) \frac{y^{2}}{2 A^{2}}+\frac{\mu_{3}(0)}{6 \sigma^{3}(0)} H_{2}(y)\right] \phi(y),
$$

where $H_{2}(y)=y^{2}-1$ is the second order Hermite polynomial, and $\Phi, \phi$ denote the standard normal distribution function and density function, respectively.

Next we state the result on bootstrapping $\bar{\beta}_{n}$ under model (1.1). Assume that $x_{1}, \ldots, x_{n}$ are all positive or all negative. As indicated above, we will denote the bootstrap version of $\bar{\beta}_{n}$ by $\beta_{n}^{*}$. Also, we will write $P_{n}$ and $E_{n}$ respectively for the conditional probability and the conditional expectation, given the data $Y_{1}, \ldots, Y_{n}$.

THEOREM 2.2. Assume that the Conditions (A.1) - (A.4) and (R.2) hold and that for every $c>0, \sum_{n=1}^{\infty} \exp \left(-c p_{n}^{2} / a_{n}^{2}\right)<\infty$. If the bootstrapped estimator $\beta_{n}^{*}$ is defined by (1.3) and $F_{n}$ by (1.5), then

(i) $\sup _{y \in \mathbb{R}}\left|P_{n}\left(a_{n} A_{n}\left(\beta_{n}^{*}-\bar{\beta}_{n}\right) \leq y\right)-\hat{\xi}_{3 n}(y)\right|=o\left(d_{3 n}\right) \quad$ a.s. and

(ii) $\sup _{y \in \mathbb{R}}\left|P_{n}\left(a_{n} A_{n}\left(\beta_{n}^{*}-\bar{\beta}_{n}\right) \leq y\right)-P\left(a_{n} A\left(\bar{\beta}_{n}-\beta\right) \leq y\right)\right|=o\left(d_{3 n}\right) \quad$ a.s. where $\hat{\xi}_{3 n}(y)$ is given by

$$
\hat{\xi}_{3 n}(y)=\Phi(y)-d_{1 n}\left[\left(\frac{m_{n}^{\prime \prime}(0)}{s_{n}(0)}-\frac{m_{n}^{\prime}(0) V^{*^{\prime}}(0)}{s_{n}^{3}(0)}\right) \frac{y^{2}}{2 A_{n}^{2}}+\frac{m_{3 n}(0)}{6 s_{n}^{3}(0)} H_{2}(y)\right] \phi(y),
$$

with $m_{n}(x)=E_{n} \psi\left(\epsilon_{1}^{*}-x\right), V^{*}(x)=s_{n}^{2}(x)=E_{n}\left(\psi\left(\epsilon_{1}^{*}-x\right)-m_{n}(x)\right)^{2}, A_{n}=$ $-m_{n}^{\prime}(0) / s_{n}(0)$, and $m_{3 n}(x)=E_{n}\left(\psi\left(\epsilon_{1}^{*}-x\right)-m_{n}(x)\right)^{3}, x \in \mathbb{R}$.

\section{Proofs}

Some more notation will be introduced first. For $x, y$, and $t$ in $\mathbb{R}$, let

$$
\begin{aligned}
S_{n}(t) & =\sum_{j=1}^{n} x_{j} \psi\left(Y_{j}-x_{j} t\right), \\
\mu_{n}(y) & =E\left[S_{n}\left(\beta+\frac{y}{a_{n} A}\right)\right], \\
V_{n}(y) & =\tau_{n}^{2}(y)=\operatorname{Var}\left(S_{n}\left(\beta+\frac{y}{a_{n} A}\right)\right), \\
\rho_{3}(y) & =E\left|\psi\left(\epsilon_{1}-y\right)-\mu(y)\right|^{3}, \\
\mu_{3 n}(y) & =E\left(S_{n}\left(\beta+\frac{y}{a_{n} A}\right)-\mu_{n}(y)\right)^{3},
\end{aligned}
$$




$$
\begin{aligned}
K_{3 n}(y, x) & =\Phi(x)-\left(\frac{\mu_{3 n}(y)}{6 \tau_{n}^{3}(y)}\right) H_{2}(x) \phi(x), \\
\gamma_{3 n}(y, t) & =\left[1+\left(\frac{\mu_{3 n}(y)}{6 \tau_{n}^{3}(y)}\right)(i t)^{3}\right] \exp \left(-t^{2} / 2\right), \\
Q_{n}\left(y, t / \tau_{n}(y)\right) & =E \exp \left(i t\left(S_{n}\left(\beta+\frac{y}{a_{n} A}\right)-\mu_{n}(y)\right) / \tau_{n}(y)\right), \\
v_{n}\left(y, t / \tau_{n}(y)\right) & =\log Q_{n}\left(y, t / \tau_{n}(y)\right)+t^{2} / 2, \\
w_{j}(y, t) & =E \exp \left(i t x_{j}\left(\psi\left(\epsilon_{1}-y\right)-\mu(y)\right) .\right.
\end{aligned}
$$

PROOF OF THEOREM 2.1. Notice that by the non-decreasing property of $\psi$ and the definition of $\bar{\beta}_{n}$,

$$
\begin{aligned}
& P\left(S_{n}(t)<0\right) \leq P\left(\bar{\beta}_{n} \leq t\right) \leq P\left(S_{n}(t) \leq 0\right), \\
& P\left(S_{n}(t)>0\right) \leq P\left(\bar{\beta}_{n} \geq t\right) \leq P\left(S_{n}(t) \geq 0\right) .
\end{aligned}
$$

Consequently, for any $y \in \mathbb{R}$,

$$
P\left(a_{n} A\left|\bar{\beta}_{n}-\beta\right|>y\right) \leq P\left(S_{n}\left(\beta+\frac{y}{a_{n} A}\right) \geq 0\right)+P\left(S_{n}\left(\beta-\frac{y}{a_{n} A}\right) \leq 0\right) .
$$

Next note that by (R.1) and (A.3), there exist constants $c_{1}>0, \eta_{1}>0$ such that $|\mu(x)| \geq c_{1}|x|$ for all $|x|<\eta_{1}$.

Now using Lemma 4.2 of Fuk and Nagaev [7], one can show that for all $n$ with $M_{n} b_{n} \leq \eta_{1} a_{n}$, and for any $c>0$,

$$
P\left(a_{n} A\left|\bar{\beta}_{n}-\beta\right|>c b_{n}\right) \leq D d_{3 n} b_{n}^{-3}
$$

where $D$ is a constant depending only on $c$. By (3.1) and (3.2), it follows that an Edgeworth expansion for $P\left(a_{n} A\left(\bar{\beta}_{n}-\beta\right) \leq y\right)$ can be obtained by finding an expansion for $P\left(S_{n}\left(\beta+y / a_{n} A\right) \leq 0\right),|y| \leq c b_{n}$, and then appraising

$$
\sup \left\{P\left(S_{n}\left(\beta+y / a_{n} A\right)=0\right):|y|=c b_{n}\right\},
$$

for some $c>0$. To that end, we use Esséen's lemma. By (R.1), for a given constant $\eta>0$, there exists a constant $b>0$, such that for all $n>b$ and $|y| \leq b_{n}$, $24\left|K_{3 n}^{\prime}(y, x)\right|<b \eta$. Fix $y \in \mathbb{R}$ with $|y| \leq b_{n}$. Then, by Esséen's Lemma (cf. [5, Lemma 16.3.2]) with $a=b / d_{3 n}$,

$$
\begin{array}{r}
\mid P\left(\left(S_{n}\left(\beta+\frac{y}{a_{n} A}-\mu_{n}(y)\right) / \tau(y) \leq x\right)-K_{3 n}(y, x) \mid\right. \\
\leq \int_{-a}^{a}\left|Q_{n}\left(y, \frac{t}{\tau_{n}(y)}\right)-\gamma_{3 n}(y, t)\right||t|^{-1} d t+\eta d_{3 n}
\end{array}
$$


The integral on the right hand side will be estimated in two parts; one ranging over $|t|<\delta a_{n} / M_{n}$ and the other ranging over $\delta a_{n} / M_{n}<|t|<a$, where the constant $\delta>0$ will be specified later. We claim that over the region $|t|<\delta a_{n} / M_{n}$, the integral on the right hand side of (3.3) can be bounded by

$$
\int_{|t|<\delta a_{n} / M_{n}} D\left(\left(|t|^{3} d_{2 n} a_{n}^{4} / \tau_{n}^{4}(y)\right)+|t|^{5}\left(d_{1 n} a_{n}^{3} / \tau_{n}^{3}(y)\right)^{2}\right) e^{-t^{2} / 2} d t=o\left(d_{3 n}\right)
$$

where $D$ is a positive constant.

To prove this, note that by Conditions (R.1) and (A.4), and by inequality 26.4 of Billingsley [3], we can find $\delta>0$ and $n_{1} \geq 1$ such that for all $n \geq n_{1}, x_{j} y /\left(a_{n} A\right) \in N$ and

$$
\sup \left\{\left|w_{j}\left(x_{j} y /\left(a_{n} A\right), t / \tau_{n}(y)\right)-1\right|:|t|<\delta a_{n} / M_{n}\right\}<1,
$$

uniformly in $j \in\{1, \ldots, n\}$. Therefore, Taylor's expansion of $\log w_{j}\left(x_{j} y / a_{n} A, t / \tau_{n}(y)\right)$ around $t=0$, inequality 16.2 .8 of Feller [8], and the facts $d_{1 n}^{2}=0\left(d_{3 n}^{2}\right)$ and $d_{2 n}^{2}=o\left(d_{3 n}^{2}\right)$ yield (3.4) after some simplifications.

Next we estimate the integral (3.3) over $\delta a_{n} / M_{n}<|t|<a$. Obviously the integral on the right hand side is smaller than

$$
\int_{\delta a_{n} / M_{n}<|t|<a}\left|Q_{n}\left(y, t / \tau_{n}(y)\right)\right||t|^{-1} d t+\int_{\delta a_{n} / M_{n}<|t|<a}\left|\gamma_{3 n}(y, t)\right||t|^{-1} d t .
$$

Using [2, Theorem 8.9], and the fact that for $n$ large, $\tau_{n}(y) \geq c^{2} a_{n}^{2}$ for some $c>0$ uniformly in $|y| \leq b_{n}$, we get

$$
\left|Q_{n}\left(y, t / \tau_{n}(y)\right)\right| \leq e^{-\delta|t|^{2} / 3}
$$

for all $|t| \leq D / d_{3 n}$, where $D>0$ is a constant, not depending on $y$ and $n$.

Next define

$$
Z_{j}=\frac{x_{j}}{\tau_{n}(y)}\left(\psi\left(\epsilon_{j}-\frac{x_{j} y}{a_{n} A}\right)-\mu\left(\frac{x_{j} y}{a_{n} A}\right)\right), \quad j=1, \ldots, n .
$$

Then as in the proof of $[2$, Theorem 8.9], we have

$$
\left|E e^{i t Z_{j}}\right|^{2} \leq \exp \left(\frac{\left|t x_{j}\right|^{2}}{\tau_{n}^{2}(y)}\left[\sigma^{2}\left(\frac{x_{j} y}{a_{n} A}\right)-\frac{\left|t x_{j}\right|}{\tau_{n}(y)} \rho_{3}\left(\frac{x_{j} y}{a_{n} A}\right)\right]\right)
$$

for all $t \in \mathbb{R}$. Since $Q_{n}\left(y, t / \tau_{n}(y)\right)=\prod_{j=1}^{n} E \exp \left(i t Z_{j}\right)$, applying Condition (A.2) together with the bound (3.6) over the region $D / d_{3 n}<|t|<b / d_{3 n}$, and using (3.5) over $\delta a_{n} / M_{n}<|t| \leq D / d_{3 n}$, we get

$$
\int_{\delta a_{n} / M_{n}<|t|<b / d_{3 n}}\left|Q_{n}\left(y, t / \tau_{n}(y)\right)\right||t|^{-1} d t=o\left(d_{3 n}\right) .
$$


Also, note that by the Mill's ratio, $\int_{|t|>\delta a_{n} / M_{n}}\left|\gamma_{3 n}(y, t)\right||t|^{-1} d t=o\left(d_{3 n}\right)$. Therefore, we have shown that

$$
\sup _{|y| \leq b_{n}} \sup _{x \in \mathbb{R}}\left|P\left(\left(S_{n}\left(\beta+\frac{y}{a_{n} A}\right)-\mu_{n}(y)\right) / \tau(y) \leq x\right)-K_{3 n}(y, x)\right|=o\left(d_{3 n}\right) .
$$

Now replacing $x$ by $-\mu_{n}(y) / \tau_{n}(y)$, we get

$$
\sup _{|y| \leq b_{n}}\left|P\left(a_{n} A\left(\bar{\beta}_{n}-\beta\right) \leq y\right)-K_{3 n}\left(y,-\mu_{n}(y) / \tau_{n}(y)\right)\right|=o\left(d_{3 n}\right) .
$$

Next using Taylor's expansion, together with the inequality: for $k \geq 0, D>0$, $|\theta| \leq D$, and $|y| \leq b_{n}, \phi^{(k)}\left(y+\theta y^{2} d_{3 n}\right) \leq D\left(1+|y|^{k}\right) \phi(y)$, one can show that

$$
\begin{aligned}
K_{3 n}(y, & \left.-\mu_{n}(y) / \tau_{n}(y)\right) \\
= & \Phi(y)-d_{1 n}\left[\left(\frac{\mu^{\prime \prime}(0)}{\sigma(0)}-\frac{V^{\prime}(0) \mu^{\prime}(0)}{\sigma^{3}(0)}\right) \frac{y^{2}}{2 A^{2}}+\frac{\mu_{3}(0)}{6 \sigma^{3}(0)} H_{2}(y)\right] \phi(y)+o\left(d_{3 n}\right) \\
= & \xi_{3 n}(y)+o\left(d_{3 n}\right),
\end{aligned}
$$

uniformly over $|y| \leq b_{n}$. This gives the expansion for $P\left(a_{n} A\left(\bar{\beta}_{n}-\beta\right) \leq y\right)$ for $|y| \leq b_{n}$. In view of (3.2), it follows that

$$
\sup _{y \in \mathbb{R}}\left|P\left(a_{n} A\left(\bar{\beta}_{n}-\beta\right) \leq y\right)-\xi_{3 n}(y)\right|=o\left(d_{3 n}\right),
$$

which completes the proof of Theorem 2.1 .

Next we give a proof of Theorem 2.2. The notation will be the same as in the proof of Theorem 2.1 with the convention that we will put an asterix over a letter to denote its bootstrap counterpart. The following lemma will be used repeatedly.

LEMMA 3.1. Suppose that the conditions of Theorem 2.2 are satisfied. Let $F_{n}$ be the resampling distribution defined as in (1.5). Then:

(i) For any $M>0$,

$$
\sup \left\{\left|w_{n}(y, t)\right|:|t| \leq M,|y| \leq M\right\}=o(1) \quad \text { a.s. }
$$

where $w(y, t)=E_{n}\left[\exp \left(i t \psi\left(\epsilon_{1}^{*}-y\right)\right)\right]$.

(ii) For $h$ a function with a bounded first derivative, and for any $M>0$,

$$
\sup \left\{\left|E_{n} h\left(\epsilon_{1}^{*}-y\right)-E h\left(\epsilon_{1}-y\right)\right|:|y| \leq M\right\}=o(1) \quad \text { a.s. }
$$

Lemma 3.1 can be proved using assumption (R.2), [7, Lemma 4.2] and a discretizing argument as in the proof of [1, Lemma 4.2]. We omit the details. 
PROOF OF THEOREM 2.2. The first part of the theorem can be proved by tracking the proof of Theorem 2.1 ; nevertheless, it must be considered in the almost sure sense. Note that by (A.3), (R.2), the monotonicity of $\psi$ (cf. (3.1)), and Lemma 3.1, there exist constants $c>0, \eta_{1}>0$ such that for all large $n$,

$$
\left|m_{n}(x)\right|>c|x| \text { for all }|x| \leq \eta_{1}, \quad \text { a.s. }
$$

and

$$
P_{n}\left(\left|\beta_{n}^{*}-\bar{\beta}_{n}\right|>u\right) \leq \exp \left(-c u^{2} a_{n}^{2}\right) \quad \text { a.s. }
$$

for any $u>0$. Consequently,

$$
P_{n}\left(\left|a_{n} A_{n}\left(\beta_{n}^{*}-\bar{\beta}_{n}\right)\right| \geq b_{n}\right) \leq a_{n}^{-3} \quad \text { a.s. }
$$

Given any $\eta>0$, by Lemma 3.1 , there exists $b^{*}>0$ (possibly random) such that for all $n>b^{*},|y| \leq b_{n}$, and $x \in \mathbb{R}, 24\left|K_{3 n}^{*}(y, x)\right| \leq b^{*}$ a.s. Hence writing $a=b^{*} / d_{3 n}$, by Esséen's Lemma,

$$
\begin{gathered}
\left|P_{n}\left(\left(S_{n}^{*}\left(\bar{\beta}_{n}+\frac{y}{a_{n} A_{n}}\right)-m_{n}(y)\right) / \tau_{n}^{*}(y) \leq x\right)-K_{3 n}^{*}(y, x)\right| \\
\quad \leq \int_{-a}^{a}\left|Q_{n}^{*}\left(y, t / \tau_{n}^{*}(y)\right)-\gamma_{3 n}^{*}(y, t)\right||t|^{-1} d t+\eta d_{3 n} .
\end{gathered}
$$

Now, using Lemma 3.1, Conditions (A.2) and (R.2), and retracing the proof of Theorem 2.1, it can be shown (see Karabulut [9]) for details) that

$$
\int_{|t| \leq b d_{3 n}^{-1}}\left|Q_{n}^{*}\left(y, \frac{t}{\tau_{n}^{*}(y)}\right)-\gamma_{3 n}^{*}(y, t)\right||t|^{-1} d t=o\left(d_{3 n}\right) \quad \text { a.s. }
$$

Hence, we have

$$
\sup _{|y| \leq b_{n}} \sup _{x \in \mathbb{R}}\left|P_{n}\left(\left(S_{n}^{*}\left(\bar{\beta}_{n}+\frac{y}{a_{n} A_{n}}\right)-m_{n}(y)\right) / \tau_{n}^{*}(y) \leq x\right)-K_{3 n}^{*}(y, x)\right|=o\left(d_{3 n}\right) \quad \text { a.s. }
$$

Next using bootstrap analogs of inequality (3.1) and setting $x=-\mu_{n}^{*}(y) / \tau_{n}^{*}(y)$ in $K_{3 n}^{*}(y, x)$, and then expanding the coefficients in Taylor's series, the last result becomes

$$
\sup _{|y| \leq b_{n}}\left|P_{n}\left(a_{n} A_{n}\left(\beta_{n}^{*}-\bar{\beta}_{n}\right) \leq y\right)-\hat{\xi}_{3 n}(y)\right|=o\left(d_{3 n}\right) \quad \text { a.s. }
$$

Finally, because of inequality (3.6), the first part of Theorem 2.2 is proved.

To prove the second part of the theorem, note that by the first part and Theorem 2.1 , it is enough to show that

$$
\sup _{y \in \mathbb{R}}\left|\hat{\xi}_{n}(y)-\xi_{n}(y)\right|=o\left(d_{3 n}\right) \quad \text { a.s.. }
$$

Repeated use of Lemma 1 proves this. Therefore, the proof of Theorem 2.2 is complete. 


\section{References}

[1] G. J. Babu, and K. Singh, 'On one term Edgeworth correction by Efron's bootstrap', Sankhyā Ser. A 46 (1984), 219-232.

[2] R. N. Bhattacharya, and R. Ranga Rao, Normal approximation and asymptotic expansions (R. E. Krieger Publishing Co., Malabar, 1986).

[3] P. Billingsley, Probability and measure (Wiley, New York, 1986).

[4] B. Efron, 'Bootstrap methods: another look at the jackknife', Ann. Statist. 7 (1979), 1-26.

[5] W. Feller, An introduction to probability theory and its applications, Vol. 2, 2nd edition (Wiley, New York, 1971).

[6] D. A. Freedman, 'Bootstrapping regression models', Ann. Statist. 9 (1981), 1218-1228.

[7] D. K. Fuk, and S. V. Nagaev, 'Probability inequalities for sums of independent random variables', Theor. Prob. Appl. 16 (1971), 643-660.

[8] P. J. Huber, 'Robust regression: asymptotics, conjectures and Monte Carlo', Ann. Statist. 1 (1973), 799-821.

[9] E. Karabulut, Edgeworth expansion and bootstrap approximation for the distribution of $M$ estimators of a simple linear regression parameter without Cramer's Condition (M. S. thesis, Department of Statistics, Iowa State University, 1991).

[10] S. N. Lahiri, 'Bootstrapping M-estimators of a multiple linear regression parameters', Ann. Statist. 20 (1992), 1548-1570.

[11] W. Navidi, 'Edgeworth expansions for bootstrapping regression models', Ann. Statist. 17 (1989), 1472-1478.

[12] M. B. Qumsiyeh, 'Edgeworth expansion in regression models', J. Multivariate Anal. 17 (1990), 1472-1478.

[13] J. T. Ringland, 'Robust multiple comparisons', J. Amer. Statist. Assoc. 78 (1983), 145-151.

[14] G. Shorack, 'Bootstrapping robust regression', Comm. Statist. Theory Methods 11 (1982), 961-972.

[15] K. Singh, On the asymptotic accuracy of Efron's bootstrap, Ann. Statist. 6 (1981), 1187-1195.

[16] A. M. Tiro, Edgeworth expansion and bootstrap approximation for $M$-estimators of linear regression parameters with increasing dimensions (Ph. D. dissertation, Department of Statistics, Iowa State University, 1991).

Department of Statistics

Gazi University

Ankara

Turkey
Department of Statistics Iowa State University Ames, Iowa 50011 